\title{
Correspondence Between Psychometric and Clinical High Risk for Psychosis in an Undergraduate Population
}

\author{
David C. Cicero \\ University of Hawai'i at Manoa
}

Theresa M. Becker

University of Washington

\author{
Elizabeth A. Martin \\ VA Pittsburgh Healthcare System, Pittsburgh, Pennsylvania
}

\author{
Anna R. Docherty \\ Virginia Commonwealth University
}

\author{
John G. Kerns \\ University of Missouri
}

\begin{abstract}
Despite the common use of either psychometric or clinical methods for identifying individuals at risk for psychosis, previous research has not examined the correspondence and extent of convergence of these 2 approaches. Undergraduates $(n=160)$, selected from a larger pool, completed 3 self-report schizotypy scales: the Magical Ideation Scale, the Perceptual Aberration Scale, and the Revised Social Anhedonia Scale. They were administered the Structured Interview for Prodromal Syndromes. First, high correlations were observed for self-report and interview-rated psychotic-like experiences ( $r$ s between .48 and $.61, p<.001)$. Second, $77 \%$ of individuals who identified as having a risk for psychosis with the self-report measures reported at least 1 clinically meaningful psychotic-like experience on the Structured Interview for Prodromal Syndromes. Third, receiver operating characteristic curve analyses showed that the self-report scales can be used to identify which participants report clinically meaningful positive symptoms. These results suggest that mostly White undergraduate participants who identify as at risk with the psychometric schizotypy approach report clinically meaningful psychotic-like experiences in an interview format and that the schizotypy scales are moderately to strongly correlated with interview-rated psychotic-like experiences. The results of the current research provide a baseline for comparing research between these 2 approaches.
\end{abstract}

Keywords: schizotypy, psychometric high risk, clinical high risk, psychotic-like experiences, prodrome

Schizophrenia is a debilitating mental illness with an onset typically in late adolescence or early adulthood (Kendler, Tsuang, \& Hays, 1987). Two factors that affect the course of the illness are age of onset (Rabinowitz, Levine, \& Hafner, 2006) and duration of untreated psychosis (DUP; i.e., length of time between the onset of psychosis and first treatment; Norman, Lewis, \& Marshall, 2005). Early onset and longer DUP are predictive of poor prognosis including lower overall quality of life, worse social functioning,

This article was published Online First April 7, 2014.

David C. Cicero, Department of Psychology, University of Hawai'i at Manoa; Elizabeth A. Martin, Mental Illness Research, Education, and Clinical Center, VA Pittsburgh Healthcare System, Pittsburgh, Pennsylvania; Theresa M. Becker, Institute for Learning and Brain Sciences, University of Washington; Anna R. Docherty, Department of Statistical Genetics, Virginia Institute for Psychiatric and Behavioral Genetics, Virginia Commonwealth University; John G. Kerns, Department of Psychological Sciences, University of Missouri.

Work on this article was supported by National Institute of Mental Health Grant MH086190.

Correspondence concerning this article should be addressed to David C. Cicero, University of Hawai'i at Manoa, Department of Psychology, Sakamaki Hall, C400, 2530 Dole Street, Honolulu, HI 96822. E-mail: dcicero@ hawaii.edu and increased symptoms (Levine \& Rabinowitz, 2009; Marshall et al., 2005). Moreover, many people with schizophrenia continue to experience these poor outcomes despite receiving state-of-the-art treatment (Addington, Leriger, \& Addington, 2003). Early identification and treatment of people at risk for the development of schizophrenia may delay the onset of the disorder, reduce DUP, and potentially prevent the onset of the disorder altogether (Addington, Epstein, et al., 2011; Melle et al., 2008). Thus, research on the identification of people at risk for schizophrenia holds promise in improving the lives of individuals with a liability for schizophrenia.

Risk for schizophrenia is generally described as two phases: premorbid and prodromal (Keshavan et al., 2009). The premorbid phase describes an individual's level of functioning from birth until the onset of attenuated symptoms and may include mild impairments in social functioning, subclinical perceptual aberrations, magical ideation, and cognitive limitations (Stoffelmayr, Dillavou, \& Hunter, 1983). The prodromal phase is characterized by marked changes in mental state in which changes appear from the individual's premorbid functioning prior to the emergence of frank psychosis (Yung \& McGorry, 1996). Most people who develop schizophrenia report a prodromal phase that lasts for weeks or months and includes increasing attenuated symptoms of psychosis, such as delusion-like and hallucination-like experiences 
(Klosterkötter, Hellmich, Steinmeyer, \& Schultze-Lutter, 2001). Finally, the acute phase of the illness involves frank psychosis, characterized by the presence of delusions, hallucinations, and disorganization (Yung, 2003). Both the premorbid and prodromal phases of schizophrenia represent functioning prior to the onset of or conversion to psychosis.

Researchers studying risk for psychosis have taken several approaches to identifying people who may be at risk. Two common approaches are the psychometric high-risk strategy (i.e., schizotypy; Chapman, Chapman, Raulin, \& Edell, 1978; Lenzenweger, 1994; Meehl, 1962) and the clinical high-risk approach (Addington et al., 2007; Addington \& Heinssen, 2012; Cannon et al., 2008; T. J. Miller et al., 2003). Schizotypy refers to traits or symptoms similar to schizophrenia but in a diminished form, and schizotypy reflects a liability for the development of schizophrenia (Chapman, Chapman, Raulin, \& Edell, 1978; Meehl, 1962). Schizotypy research aims to provide insight into the symptoms of schizophrenia while removing confounds associated with patient research, such as medication (Neale \& Oltmanns, 1980). The psychometric schizotypy approach usually identifies people with schizotypy by selecting participants with sex-normed $z$ scores greater than 1.96 (i.e., the upper $2.5 \%$ of the distribution) on a combination of the Revised Social Anhedonia Scale (SocAnh; Eckblad, Chapman, Chapman, \& Mishlove, 1982) as a "negative schizotypy group" and the Perceptual Aberration Scale (PerAb; Chapman, Chapman, \& Raulin, 1978) and Magical Ideation Scale (MagicId; Eckblad \& Chapman, 1983) as a "positive schizotypy group." Together, these scales are referred to as the Wisconsin Schizotypy Scales. Participants in psychometric schizotypy studies are generally population-based samples, often selected from a pool of undergraduate students (e.g., Chapman, Chapman, Kwapil, Eckblad, \& Zinser, 1994; Gooding, Tallent, \& Matts, 2005; Kwapil, Miller, Zinser, Chapman, \& Chapman, 1997). Previous longitudinal studies have found that around five percent of people identified as psychometric schizotypes demonstrate a psychotic disorder at a 10-year follow-up and that this rate increases to nearly $40 \%$ in people with both high negative and positive schizotypy (Chapman et al., 1994). Critically, this strategy aims to identify people in the premorbid phase, who are usually not "help-seeking" in that they are not referred for participation based on seeking treatment in a psychological clinic.

The fifth edition of the Diagnostic and Statistical Manual of Mental Disorders includes attenuated psychosis syndrome in Section III as a condition in need of further research (American Psychiatric Association, 2013). The DSM workgroup considered including attenuated psychosis syndrome in the psychotic disorders section but ultimately decided to include it in Section III, in part due to assessment concerns (Tandon, Shah, Keshavan, \& Tandon, 2012; Woods, Walsh, Saksa, \& McGlashan, 2010). In North America, the primary tool used to assess risk in this approach is the Structured Interview for Prodromal Syndromes (SIPS; T. J. Miller et al., 2003). Thus, it can be useful to examine the psychometric properties of this assessment, even in nonclinical populations. In clinical populations, this approach has yielded accuracy estimates in predicting who will "convert to psychosis" of up to $35 \%$ (Cannon et al., 2008), which is higher than the 5\% reported in schizotypy research in undergraduate samples. Individuals who are identified as having attenuated psychosis syndrome may not develop schizophrenia, but they may be more vulnerable than others to developing other psychiatric conditions (Addington, Cornblatt, et al., 2011; Tandon, Shah, et al., 2012; Woods et al., 2010)

Despite the high volume of influential research resulting from both of these traditions, few studies have offered a way to compare and contrast these results. Research comparing these two approaches is important because the efficacy of the psychometric schizotypy approach is predicated on two suppositions. First, psychometric schizotypy researchers hypothesize that people identified as being at risk for psychosis are having clinically meaningful psychotic-like experiences that can be used to model full-blown psychotic symptoms. Second, schizotypy researchers hypothesize that people identified with the psychometric approach are at risk for developing psychosis.

Our ultimate goal in the current research is to provide a metric for comparing the results of research from the psychometric schizotypy approach to results from the clinical high-risk approach. Our first goal in the current research is to provide further validation to the schizotypy scales by examining the percentage of people identified as at risk who experience clinically meaningful attenuated positive symptoms as assessed by the SIPS. As mentioned, one major goal of schizotypy research is to model symptoms of schizophrenia without the confounds of schizophrenia research, such as medication (Neale \& Oltmanns, 1980). The efficacy of this approach depends on similarities between schizotypy symptoms and psychotic symptoms. Thus, a question faced by schizotypy researchers is whether participants who are recruited from undergraduate populations have clinically significant psychotic-like experiences. A reader would be hard pressed to find a journal article on schizotypy that does not explicitly address this issue in the discussion section. Despite this interest, few studies have examined whether people with self-reported psychometric schizotypy report psychotic-like experiences on interview measures of psychotic-like experiences, and no studies have examined this with the SIPS in the undergraduate samples common in schizotypy research.

Our second goal in the current research is to compare participants identified as at risk in these two approaches. The questionnaires utilized by the psychometric approach have been used by schizotypy researchers for decades (Chapman, Chapman, Raulin, \& Edell, 1978; Edell, 1995). In contrast, the SIPS is a relatively new measure of psychosis risk (T. J. Miller et al., 2002). The SIPS has been referred to as the gold standard in psychosis risk assessment (Kline et al., 2012) and is currently being used in high-profile research programs that are making rapid progress in the understanding of psychosis risk (Addington et al., 2007, 2012). Thus, a goal in the current research is to examine the correspondence between participants identified as at risk in the psychometric high-risk approach with those identified as high risk with the clinical high-risk approach.

As described above, the most commonly used psychometric schizotypy scales, the Wisconsin Schizotypy Scales, were developed at the University of Wisconsin with undergraduate research participants (Chapman, Chapman, \& Raulin, 1978; Eckblad \& Chapman, 1983; Eckblad et al., 1982). Many of the original studies on the psychometric properties and construct validity of the scales, including longitudinal studies measuring the development of schizophrenia, contained primarily undergraduate students (Chapman et al., 1994; Gooding et al., 2005; Kwapil, 1998; Kwapil et al., 
1997). Many researchers have carried on this tradition with undergraduate participants, and college students are commonly sampled in productive schizotypy research programs. However, few recent studies have examined whether college student participants experience clinically significant attenuated psychotic symptoms. We chose undergraduate students, as opposed to young adults from the community, as participants because a major goal of the research was to provide a basis for a comparison between psychometric schizotypy research and clinical high-risk work.

Although the correspondence between the Wisconsin Schizotypy Scales and the SIPS has not been examined, researchers have examined the correspondence between these scales and other interview measures of psychotic-like experiences. For example, the Chapmans and their colleagues developed the Wisconsin Manual for Assessing Psychotic-Like Experiences (Chapman \& Chapman, 1980; see Kwapil, Chapman, \& Chapman, 1999, for a review). Estimates of the percentage of participants with psychometric schizotypy who reported clinically meaningful psychotic-like experiences on the Wisconsin Manual range from 59 to $60 \%$ for people with deviant PerAb scores (i.e., $>1.96$ SDs above the mean on PerAb; Allen, Chapman, Chapman, Vuchetich, \& Frost, 1987; Chapman \& Chapman, 1980), from 42 to $45 \%$ for people with deviant PerMag scores (i.e., $>$ a combined $3 S D$ above the mean on PerAb and MagicId), and 54\% for people with deviant MagicId scores (i.e., $>1.96 S D$ above the mean on MagicID; Eckblad \& Chapman, 1983). In addition, at least two studies have used the Wisconsin Schizotypy Scales and the SIPS in a single study (Delawalla et al., 2006; Tandon, Montrose, et al., 2012). However, no studies have reported correlations between Wisconsin Schizotypy Scales scores and SIPS ratings or whether people with high psychometric schizotypy report clinically meaningful attenuated positive symptoms and meet criteria for clinical high risk. To allow meaningful comparisons between results of psychometric and clinical high-risk studies, researchers must compare and contrast the results of the most commonly used instruments in a single sample.

As mentioned, our first goal in the current research was to examine the percentage of individuals identified as at risk with the psychometric schizotypy approach who are actually experiencing clinically significant psychotic-like symptoms. We expected to find the following: (a) a positive correlation between positive schizotypy scores and interview-rated psychotic-like symptoms; (b) the positive schizotypy group would have higher positive symptom ratings than the negative and psychometric control groups, while the negative schizotypy group would have higher negative symptom ratings than the positive and psychometric control groups; (c) a higher percentage of participants with psychometrically defined positive schizotypy than participants with negative schizotypy or psychometric control participants would report clinically meaningful attenuated psychotic-like experiences on the structured interview; and (d) high sensitivity and specificity of the psychometric schizotypy scales in predicting which participants would experience clinically meaningful psychotic-like experiences. Our second goal in the current research was to examine what percentage of people identified as at risk with the psychometric approach would also be identified as at risk with the clinical high-risk approach. Given the criteria of recent onset, frequent symptoms, and/or recent drop in global assessment of functioning scores in determining clinical high risk, we did not expect to find many participants meeting these risk categories in our undergrad- uate sample. However, we expected to find that a high percentage of participants meeting clinical high-risk criteria would also meet psychometric high-risk criteria.

\section{Method}

\section{Participants}

Participants were recruited following the psychometric high-risk approach (Lenzenweger, 1994), which involved a two-step process. First, participants were recruited from a larger pool of undergraduate students $(n=2,244)$. These participants completed abbreviated versions of the Magical Ideation Scale (Eckblad \& Chapman, 1983), the Perceptual Aberration Scale (Chapman, Chapman, \& Raulin, 1978), and the Revised Social Anhedonia Scale (Eckblad et al., 1982). These abbreviated questionnaires included 10 items from each scale that were chosen based on item-total correlations from a previous data set.

Following previous research (e.g., Chapman et al., 1994; Edell, 1995; Lenzenweger, 1994), participants were recruited to take part in the second screening phase if they scored above 1.96 sexnormed standard deviations ( $S D \mathrm{~s}$ ) above the mean on the MagicId, PerAb, or SocAnh, or if they scored a combined three sex-normed standard deviations above the mean on the MagicId and PerAb. In addition, a psychometric control group of participants scoring less than 0.5 sex-normed standard deviations above the mean on all three scales was recruited to participate in the second screening phase.

In the second screening phase, participants completed the full versions of the MagicId, PerAb, and SocAnh and were categorized into a positive, negative, or psychometric control group based on norms established in previous research (Kerns \& Berenbaum, 2003). Participants scoring 1.96 sex-normed standard deviations above the mean on the MagicId and PerAb or a combined three sex-normed standard deviations above the mean on the MagicId and PerAb were assigned to the positive schizotypy group $(n=$ 59). Participants scoring over 1.96 sex-normed standard deviations above the mean on the SocAnh were assigned to the negative schizotypy group $(n=64)$, and participants scoring below 0.5 sex-normed standard deviations above the mean on all three scales were assigned to the psychometric control group $(n=45)$. Eight participants met criteria for both the positive and negative schizotypy groups. The computer program immediately calculated participants' scores, and all participants meeting criteria for positive and negative schizotypy and one out of 10 randomly selected comparison participants were invited to participate in the second in-person session. This two-step strategy allowed us to confirm that the participants were indeed high scorers and to guard against regression to the mean.

Participants in the positive schizotypy group had a mean age of 18.56 years $(S D=0.85)$ and were $43 \%$ female, $76 \%$ White, $10 \%$ African American, 4\% Asian American, and 8\% other. Participants in the negative schizotypy group had a mean age of 18.96 years $(S D=1.53)$ and were $65 \%$ female, $72 \%$ White, $19 \%$ African American, $4 \%$ biracial, and $6 \%$ other. Participants in the psychometric control group had a mean age of 18.58 years $(S D=1.03)$ and were 54\% female, 94\% White, 2\% African American, and 2\% Asian American. 


\section{Materials}

Positive schizotypy. Magical ideation was measured with the Magical Ideation Scale (Eckblad \& Chapman, 1983). The MagicId is a 30 -item true-false scale that measures a tendency to endorse beliefs that by conventional standards are considered invalid (e.g., the government refuses to tell us the truth about flying saucers). A second measure of positive schizotypy was the Perceptual Aberration Scale (Chapman, Chapman, \& Raulin, 1978) The PerAb is a 35-item true-false scale that measures schizophrenic-like distortion in one's perception of one's own body (e.g., "I can remember times in which it seemed that one of my limbs took on an unusual shape").

The MagicId and PerAb are two of the most commonly used schizotypy measures, and they have been found to correlate with many of the same constructs as measures of positive symptoms of schizophrenia, including cognitive, emotional, and social impairment variables (Kwapil, Barrantes-Vidal, \& Silvia, 2008). Previous work has reported Cronbach's internal reliability fluctuating between .78 and .92 for these measures and test-retest reliability between .75 and .82 (Fonseca-Pedrero et al., 2008; Graves \& Weinstein, 2004; Kwapil et al., 2008). However, one criticism of the PerAb and MagicId is that some studies have reported testretest reliability of .63-.76 for PerAb and .73-.79 for MagicId (Winterstein, Ackerman, Silvia, \& Kwapil, 2011). Factor analytic studies have found that they load on a cognitive-perceptual factor along with other measures of positive schizotypy (Cicero \& Kerns, 2010; Wuthrich \& Bates, 2006). Scores on MagicId and PerAb are often combined to create a single positive schizotypy group. As such, the two scales do not have discriminant validity from each other. In the current research, MagicId had a mean of 11.49, SD of 7.48, Cronbach's alpha of .86, skewness of 0.30 , and kurtosis of -1.07 . PerAb had a mean of $8.50, S D$ of 8.18 , Cronbach's alpha of .89 , skewness of 1.12 , and kurtosis of 0.52 . We were unable to assess test-retest reliability because of the cross sectional nature of this study.

Negative schizotypy. Negative schizotypy was measured with the Revised Social Anhedonia Scale (Eckblad et al., 1982), a 40-item true-false scale that measures a lack of pleasure from social relationships and interactions (e.g., "I never really had close friends in high school"). Previous research has found that social anhedonia is predictive of future schizophrenia-spectrum disorders (Kwapil et al., 1997). Previous studies have reported internal reliability of .79 to .90 and test-retest reliability above .80 (Fonseca-Pedrero et al., 2008; Horan, Brown, \& Blanchard, 2007). In the current research, SocAnh had a mean of 12.07, $S D$ of 8.12, Cronbach's alpha of .86, skewness of 0.54 , and kurtosis of -0.72 .

Structured Interview for Prodromal Syndromes. Participants who met criteria for positive, negative, or psychometric control groups as outlined above were recruited back for a separate, more in depth, study session that took approximately 30 to 90 minutes. The Structured Interview for Prodromal Syndromes (T. J. Miller et al., 2003), a semistructured interview designed to assess the prodromal state of the development of schizophrenia, was used to assess risk for psychosis and to obtain ratings for positive, negative, and disorganized symptoms of the prodromal syndrome. The SIPS and the accompanying Scale of Prodromal Syndromes (SOPS) were designed to be similar to the Positive and Negative Syndrome Scales (PANSS; Kay, Fiszbein, \& Opler, 1987) but to assess a wider range of symptom severity that includes prodromal symptoms. The SIPS was designed to measure the three main factors of schizophrenia symptoms: positive, negative, and disorganized. The SOPS has ratings for positive symptoms including delusional ideation, paranoia, grandiosity, perceptual anomalies, and disorganized communication; negative symptoms including social anhedonia, avolition, expression of emotion, experience of emotions and self, ideational richness, and occupational functioning; and disorganized symptoms including odd behavior or appearance, bizarre thinking, trouble with focus and attention, and impairment in personal hygiene. Unlike self-report questionnaires, the SIPS enables the clinical interviewer to ask detailed questions relating to each symptom construct and to observe and rate the clinical presentation of the participant.

Prior to conducting this study, the first author trained in the administration and scoring of the interview with the developers of the SIPS/SOPS at the Psychosis Prodrome Research Clinic (PRIME Clinic) at Yale University. Previous research has found that this workshop is successful in training researchers to conduct the interview with excellent interrater reliability (T. J. Miller et al., 2002, 2003). The training workshop is a one and a half day program. First, trainees are given a lecture on the SIPS/SOPS, including its development, intended purposes, and clinical examples of symptoms and risk categories. Second, trainees view two videotaped interviews, with one patient meeting criteria for the prodrome and the other not meeting these criteria. The ratings for these patients are thoroughly discussed. Third, trainees are led in a discussion of the nuances of rating risk for psychosis, including difficult topics such as delusional conviction and differentiation of persecutory and nonpersecutory ideas of reference. Finally, participants view two new interviews with patients and are blind to the risk status of the patients. One patient is at risk for psychosis, and one patient is not. In a study with 35 different trainees who took part in six separate workshops, T. J. Miller et al. (2003) reported an average kappa of 0.86 for risk diagnoses, and intraclass correlation coefficient values greater than .75 for positive, negative, and disorganized global ratings. Certification in current workshops requires trainees to accurately identify the at-risk patient and not at-risk patient and to come within one anchor point on the $0-6$ scale for all 19 SOPS ratings. The first author met these criteria for certification in the administration of the SIPS.

\section{Procedure}

In the first screening phase, participants completed the abbreviated versions of the schizotypy scales online as part of a larger screening battery that took approximately one hour. As described above, participants scoring 1.96 sex-normed standard deviations above the mean on the PerAb or the MagicID, a combined 3.00 sex-normed $S D$ above the mean on both scales, $1.96 S D$ above the mean on the SocAnh, or less than 0.50 sex normed $S D$ above the mean on all three scales for controls were contacted via e-mail and telephone and invited to the in-person second screening phase. The second screening phase included the full versions of the schizotypy scales mixed together and called the Survey of Attitudes and Experiences as part of a larger battery. This session took place in private cubicles in the laboratory and lasted approximately one hour. The computer program immediately calculated participants' scores, and all participants meeting criteria for positive and neg- 
ative schizotypy and one out of 10 randomly selected comparison participants were invited to participate in the second in-person session. The second in-person session took place in an individual testing room and included the Structured Interview for Prodromal Syndromes. This session took between 30 minutes and 90 minutes, depending on participant responses. Of the interviews, 138 were conducted by the first author and the rest were conducted by the second, third, and fourth authors. All the interviews were videotaped, and all of the ratings were done by the first author. In all cases, the interviewer and/or rater was blind to the group membership and questionnaire scores of all participants. As can be seen in Table 1, the SIPS ratings had high interrater reliability in the current research.

The SIPS allows for diagnosis of attenuated positive symptoms prodromal syndrome (APSP), brief intermittent psychotic symptoms prodromal syndrome (BIPS), genetic risk and deterioration prodromal syndrome (GRD), schizotypal personality disorder (SPD), and psychotic syndrome. APSP, BIPS, and GRD are proposed diagnostic categories for psychosis risk. The first criterion of APSP is a SOPS P1-P5 (i.e., the positive symptom ratings) of 3-5, which represents clinically meaningful attenuated positive symptoms that do not reach a psychotic level (i.e., a rating of 6). To meet full criteria for APSP, the interviewee must have an onset of these symptoms or a worsening of the symptoms in the last 12 months and the symptoms must have occurred at least once a week in the past month. In the current research, one of the key dependent variables was whether participants met the first criterion (i.e., a clinically meaningful attenuated positive symptom). BIPS involves having a rating of 6 on a P1-P5 scale, having a symptom reach a 6 in the last 3 months, and having the symptoms be present for at least several minutes per day at least once a month. GRD involves the individual meeting criteria for SPD or having a first-degree relative with a psychotic disorder, and the individual having at least a 30\% drop in global assessment of functioning scores in the last 12 months. Psychotic syndrome involves at least one rating of 6 (i.e., psychotic) on the positive scale and either (a) symptoms that are seriously disorganizing or dangerous or (b) symptoms that occur at least one hour per day at an average frequency of 4 days per week over 1 month.

\section{Results}

\section{Zero-Order Correlations}

We first examined the correlations among the schizotypy scales and the symptom ratings. If there is a high correspondence between schizotypy measures and symptom ratings of prodromal symptoms, we would expect to see high correlations between positive schizotypy measures (i.e., the PerAb and MagicId) and positive symptoms ratings as well as high correlations between the negative schizotypy scale (i.e., the SocAnh) and negative symptom ratings on the SOPS. As can be seen in Table 1, both the PerAb and the MagicId were significantly correlated with the mean of the positive ratings as well as with all five individual ratings (Pearson $r$ s range from .33 to .61). The SocAnh was positively correlated with the overall negative symptom rating, social anhedonia, expression of emotion, and experience of self and emotion. These correlations suggest that the schizotypy scales are strongly related to symptom ratings on the SOPS.

\section{Group Comparisons of Interview-Rated Psychotic-Like Experiences}

If the psychometric schizotypy groups are valid indicators of psychotic-like experiences, we would expect to find that the positive group had more interview-rated positive symptoms than the negative and control groups. Moreover, the psychometric negative schizotypy group should have higher interview-rated negative symptoms than the positive and control groups. To test this, we ran three multivariate analyses of variance (MANOVAs) with group membership (i.e., positive, negative, and control) as a fixed-factor between-subjects effect predicting all positive, negative, and disorganized ratings. In this analysis, participants were not allowed to belong to more than one group. If participants met criteria for more than one group, they were assigned to the group for their highest $z$ score.

There was a significant multivariate main effect for predicting all five positive ratings, Wilks's $\lambda=.57, F(10,304)=9.87, p<$ $.001 ; \eta^{2}=.25$; all six negative ratings, Wilks's $\lambda=.58, F(12$, $302)=7.90, p<.001 ; \eta^{2}=.24$; and all four disorganized ratings, Wilks's $\lambda=.76, F(8,308)=5.68, p<.001 ; \eta^{2}=.13$.

Because all three of these MANOVAs were significant, we followed up the analysis with ANOVAs for each separate rating. Planned comparison $t$ tests followed up each significant-result ANOVA. We ran one-way ANOVAs with group membership (i.e., positive, negative, and control) as the between-subjects factor and all symptom ratings as the dependent variables. As can be seen in Table 2, groups differed in overall positive ratings as well as each individual group, $F \mathrm{~s}(2,161)$ between 10.71 and 39.43 , all $p \mathrm{~s}<$ $.001 ; \eta^{2}$ s between .12 and .34 . Planned $t$ tests revealed that the positive group had higher global interview-rated positive scores and higher scores on all five subscales than the psychometric control group, $t \mathrm{~s}(161)$ between 4.59 and 8.81, all $p \mathrm{~s}<.001$; Cohen's $d$ s between 0.72 and 1.39 , and higher scores than the negative group on all positive scales except disorganized communication, $t \mathrm{~s}(161)$ between 2.58 and 5.96, all $p \mathrm{~s}<.01$; Cohen's $d \mathrm{~s}$ between 0.41 and 0.94 . The negative group had higher global positive, delusional ideation, and paranoia scores than the psychometric control group, $t \mathrm{~s}(161)$ between 2.66 and 5.00, $p \mathrm{~s}<.01$; Cohen's $d$ s between 0.42 and 0.79 .

\section{Group Comparisons of Interview-Rated Negative Symptoms}

As can be seen in Table 2, groups differed in average negative symptom ratings and all individual ratings except ideational richness, which had very low levels across all groups in this undergraduate sample, $F_{\mathrm{S}}(2,161)$ between 5.95 and 45.77 , all $p \mathrm{~s}<.001$; $\eta^{2}$ s between .03 and .36. The negative group had higher scores than the psychometric control group on all negative measures except occupational functioning and ideational richness, $t \mathrm{~s}(161)$ between 3.43 and 8.87, all $p s<.003$; Cohen's $d$ s between 0.54 and 1.40. The negative group had higher scores than the positive group for global negative ratings, social anhedonia, and expression of emotion, $t \mathrm{~s}(161)$ between 3.24 and 7.15, all $p \mathrm{~s}<.002$; Cohen's $d \mathrm{~s}$ between 0.51 and 1.13. Finally, the positive group had higher negative scores than the psychometric control group on global negative scores, expression of emotion, experience of emotion and 


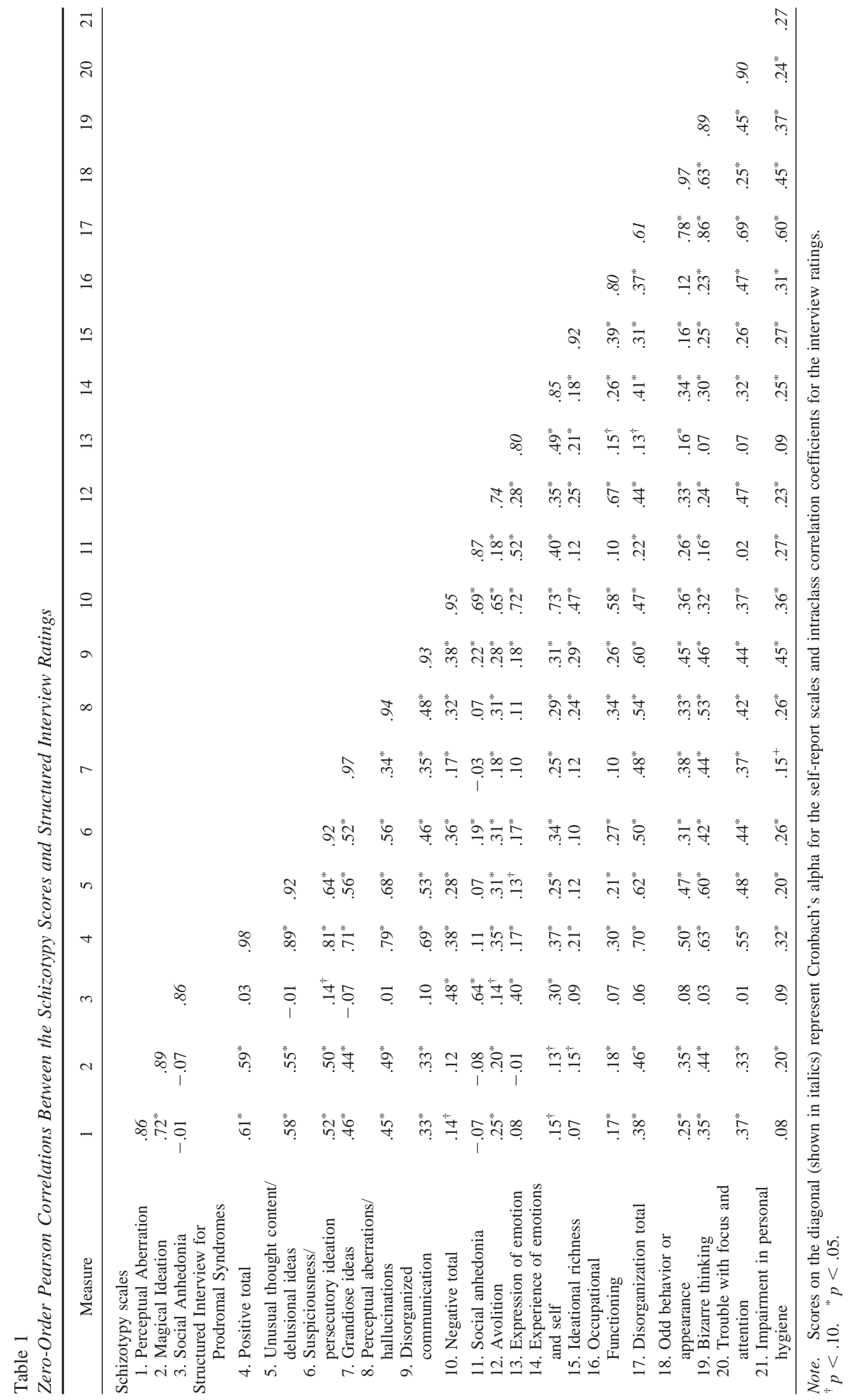


Table 2

Psychometric Schizotypy Between-Group Comparisons of Interview-Rated Symptoms

\begin{tabular}{|c|c|c|c|c|c|}
\hline Measure & $\begin{array}{l}\text { Positive group } \\
\quad M(S D)\end{array}$ & $\begin{array}{c}\text { Negative group } \\
\quad M(S D)\end{array}$ & $\begin{array}{l}\text { Control group } \\
M(S D)\end{array}$ & $\begin{array}{c}F \text { score } \\
d f(2,161)\end{array}$ & $\begin{array}{c}\text { Effect size } \\
\eta^{2}\end{array}$ \\
\hline Global positive rating & $9.93(5.27)_{\mathrm{a}}$ & $5.31(4.53)_{\mathrm{b}}$ & $2.06(3.18)_{\mathrm{c}}$ & $39.90^{*}$ & 0.34 \\
\hline Delusional ideation & $2.81(1.55)_{\mathrm{a}}$ & $1.27(1.47)_{\mathrm{b}}$ & $0.55(1.06)_{\mathrm{c}}$ & $35.35^{*}$ & 0.32 \\
\hline Paranoia & $2.07(1.13)_{\mathrm{a}}$ & $1.56(1.18)_{\mathrm{b}}$ & $0.51(0.86)_{\mathrm{c}}$ & $26.88^{*}$ & 0.26 \\
\hline Grandiosity & $1.81(1.74)_{\mathrm{a}}$ & $0.68(1.23)_{\mathrm{b}}$ & $0.32(0.63)_{\mathrm{b}}$ & $18.82^{*}$ & 0.20 \\
\hline Perceptual anomalies & $1.96(1.52)_{\mathrm{a}}$ & $0.97(1.34)_{\mathrm{b}}$ & $0.40(0.90)_{\mathrm{b}}$ & $18.96^{*}$ & 0.19 \\
\hline Disorganized communication & $1.26(1.23)_{\mathrm{a}}$ & $0.92(1.14)_{a}$ & $0.30(0.62)_{\mathrm{b}}$ & $10.70^{*}$ & 0.12 \\
\hline Global negative rating & $3.61(3.71)_{\mathrm{a}}$ & $5.73(4.19)_{\mathrm{b}}$ & $0.90(1.90)_{\mathrm{c}}$ & $25.41^{*}$ & 0.24 \\
\hline Social anhedonia & $0.50(0.91)_{\mathrm{a}}^{\mathrm{a}}$ & $2.00(1.61)_{\mathrm{b}}$ & $0.06(0.25)_{\mathrm{a}}$ & $45.77^{*}$ & 0.36 \\
\hline Avolition & $0.86(1.00)_{3}$ & $0.76(0.96)_{3}$ & $0.23(0.63)$ & $6.95^{*}$ & 0.07 \\
\hline Expression of emotion & $0.67(0.97)_{\mathrm{a}}$ & $1.24(1.22)_{\mathrm{b}}$ & $0.26(0.61)_{c}$ & $13.47^{*}$ & 0.14 \\
\hline Experience of emotion and self & $0.70(1.18)_{\mathrm{a}}$ & $1.03(1.35)_{\mathrm{a}}$ & $0.06(0.32)_{\mathrm{b}}$ & $10.80^{*}$ & 0.12 \\
\hline Ideational richness & $0.35(0.73)_{\mathrm{a}}^{\mathrm{a}}$ & $0.31(0.76)_{a}^{a}$ & $0.15(0.62)_{\mathrm{a}}$ & 1.11 & 0.01 \\
\hline Occupational functioning & $0.53(0.95)_{a}$ & $0.33(0.92)_{\mathrm{ab}}$ & $0.13(0.34)_{\mathrm{b}}$ & $3.24^{*}$ & 0.03 \\
\hline Global disorganized rating & $3.13(2.82)_{\mathrm{a}}$ & $2.05(2.30)_{\mathrm{b}}$ & $0.55(0.93)_{\mathrm{c}}$ & $17.13^{*}$ & 0.17 \\
\hline Odd behavior & $0.69(1.00)_{\mathrm{a}}$ & $0.49(0.93)_{a}$ & $0.04(0.20)_{\mathrm{b}}$ & $8.11^{*}$ & 0.09 \\
\hline Bizarre thinking & $0.91(1.26)_{\mathrm{a}}$ & $0.44(0.80)_{\mathrm{b}}^{\mathrm{a}}$ & $0.06(0.32)_{\mathrm{c}}$ & $11.29^{*}$ & 0.13 \\
\hline Focus/attention & $1.37(0.96)_{\mathrm{a}}$ & $0.89(0.86)_{\mathrm{b}}$ & $0.43(0.66)_{c}$ & $15.85^{*}$ & 0.16 \\
\hline Personal hygiene & $0.17(0.54)_{a}^{a}$ & $0.22(0.61)_{\mathrm{b}}$ & $0.02(0.15)_{\mathrm{a}}$ & 2.29 & 0.03 \\
\hline
\end{tabular}

Note. Means that share a subscript do not significantly differ from each other at $p<.05$. Positive group $=$ participants meeting criteria for high psychometric positive schizotypy; negative group = participants meeting criteria for high negative schizotypy; control group $=$ participants meeting criteria for the psychometric control group.

$* p<.05$

self, and occupational functioning, $t$ s(161) between 2.07 and 3.89 , all $p$ s $<.05$; Cohen's $d$ s between 0.33 and 0.61 .

\section{Group Comparisons of Interview-Rated Disorganized Symptoms}

As can be seen in Table 2, the groups differed in global disorganized ratings and every disorganized symptom rating except impairment in personal hygiene, $F \mathrm{~s}(2,161)$ between 8.11 and 17.13, all $p$ s $<.001 ; \eta^{2}$ s between .09 and .17. The positive group had higher global disorganized, odd behavior, bizarre thinking, and focus/attention ratings than the control group, $t \mathrm{~s}(161)$ between 3.86 and 5.72, $p$ s $<.001$; Cohen's $d$ s between 0.61 and 0.90 . The positive group had higher global disorganized, bizarre thinking, and focus/attention scores than the negative group, $t \mathrm{~s}(161)$ between 2.99 and 5.60, $p$ s $<.01$; Cohen's $d$ s between 0.47 and 0.88 . Finally, the negative group had higher scores on all of the disor- ganized ratings than the control group, $t$ s(161) between 2.07 and $3.48, p$ s $<.05$; Cohen's $d$ s between 0.33 and 0.55 .

\section{Correspondence Between Psychometric Schizotypy Groups and Interview-Rated Psychotic-Like Experiences}

First, we examined which participants met SIPS criteria for attenuated positive symptoms prodromal syndrome, brief intermittent psychotic symptoms prodromal syndrome, genetic risk and deterioration syndrome, schizotypal personality disorder, and psychotic syndrome. As can be seen in Table 3, two out 160 total participants met criteria for APSP and two participants met criteria for BIPS. All four of these participants were identified as having high positive schizotypy, and one participant met criteria for both high positive and high negative schizotypy. No participants met criteria for GRD or psychotic syndrome. Twenty-two participants

Table 3

Correspondence Between Psychometric Schizotypy Groups and SOPS Risk for Psychosis

\begin{tabular}{|c|c|c|c|c|}
\hline Risk category & $\begin{array}{l}\text { Psychometric positive } \\
n(\%)\end{array}$ & $\begin{array}{c}\text { Psychometric negative } \\
n(\%)\end{array}$ & $\begin{array}{l}\text { Psychometric control } \\
n(\%)\end{array}$ & $\begin{array}{l}\text { Total } \\
n(\%)\end{array}$ \\
\hline Attenuated positive symptoms prodromal syndrome & $2(3.4)$ & 0 & 0 & $2(1.2)$ \\
\hline $\begin{array}{l}\text { Brief intermittent psychotic symptoms prodromal } \\
\text { syndrome }\end{array}$ & 2. (3.4) & $1(1.6)$ & 0 & $2(1.2)$ \\
\hline Genetic risk and deterioration syndrome & 0 & 0 & 0 & 0 \\
\hline Psychotic syndrome & 0 & 0 & 0 & 0 \\
\hline Schizotypal personality disorder & $16(27.1)$ & $8(12.5)$ & $1(2.2)$ & $22(13.4)$ \\
\hline Total & 59 & 64 & 45 & 160 \\
\hline
\end{tabular}

Note. One person who met criteria for brief intermittent psychotic symptoms prodromal syndrome met criteria for high psychometric positive and negative schizotypy. Three participants who met criteria for schizotypal personality disorder met criteria for high psychometric positive and negative schizotypy. SOPS $=$ Scale of Prodromal Syndromes. 
met criteria for SPD. Of these 22, 16 met criteria for psychometric positive schizotypy, eight met criteria for psychometric negative schizotypy, three met criteria for both positive and negative schizotypy, and one was a psychometric control.

On the SOPS, ratings of 3 or higher represent clinically meaningful attenuated psychotic-like symptoms. One major goal of most schizotypy research is to provide insight into psychosis, and researchers have questioned how similar psychometrically identified people with schizotypy are to people who experience clinically meaningful psychotic-like symptoms. If the psychometric schizotypy scales identify people with clinically relevant symptoms, we would expect these participants to have ratings of 3 or higher on the SOPS. Thus, we created groups to mirror the psychometric schizotypy groups for interview-rated psychotic-like experiences. Participants with at least one rating of 3 or higher on any of the five positive symptom scales (i.e., delusional ideation, perceptual anomalies, paranoia, grandiosity, and disorganized communication) were assigned to the positive SIPS group. Participants with at least one negative symptom rating of 3 or higher were assigned to the negative group. Participants with no ratings over 3 were assigned to the control group. Participants who met criteria for positive and negative groups were assigned to both categories. Thus, the percentages add up to greater than 100 in Table 4.

As can be seen in Table 4, 72.9\% of participants with positive schizotypy had at least one rating of 3 or higher for at least one positive symptom on the SOPS. In other words, $73 \%$ of participants with positive schizotypy reported a clinically meaningful positive symptom on the SOPS. Similarly, over half (56.3\%) of participants with negative schizotypy were assessed with at least one clinically significant negative symptom on the SOPS, and only $22 \%$ reported no clinically significant positive or negative symptoms.

On the basis of these group classifications, we conducted sensitivity/specificity analyses for PerAb/MagicId scales "diagnosing" SIPS positive versus control group membership. Sensitivity was calculated as the number of true positives divided by the number of true positives plus the number of false negatives, and specificity was calculated as the number of true negatives divided

Table 4

Correspondence Between Psychometric Schizotypy Groups and SIPS Ratings of 3 or Higher for Positive and Negative Symptoms

\begin{tabular}{|c|c|c|c|c|}
\hline Rating & $\begin{array}{c}\text { Psychometric } \\
\text { positive } \\
n(\%)\end{array}$ & $\begin{array}{c}\text { Psychometric } \\
\text { negative } \\
n(\%)\end{array}$ & $\begin{array}{c}\text { Psychometric } \\
\text { control } \\
n(\%)\end{array}$ & $\begin{array}{l}\text { Total } \\
n(\%)\end{array}$ \\
\hline SIPS positive (3) & $43(72.9)$ & $28(43.8)$ & $4(8.9)$ & $72(42.9)$ \\
\hline SIPS negative (3) & $13(22.0)$ & $36(56.3)$ & $2(4.4)$ & $47(28.0)$ \\
\hline SIPS control (3) & $13(22.0)$ & 17 (26.6) & $39(86.7)$ & $71(42.3)$ \\
\hline Total & 59 & 64 & 45 & 160 \\
\hline
\end{tabular}

Note. Numbers in positive, negative, and total columns add up to more than $100 \%$ because some participants met criteria for the positive and the negative SIPS and/or positive and negative psychometric schizotypy groups. SIPS control (3) = no ratings of 3 or more on any of the SIPS positive and negative ratings; SIPS positive (3) = at least one rating of 3 or more on a positive SIPS item; SIPS negative (3) = at least one rating of 3 or more on a negative SIPS item. SIPS $=$ Structured Interview for Prodromal Syndromes. by the number of true negatives plus the number of false positives. Positive predictive value (PPV) is number of true positives divided by the number of true positives plus the number of false positives, and negative predictive value (NPV) is the number of true negatives divided by the number of true negatives plus the number of false negatives (Haynes, Smith, \& Hunsley, 2011). The PerAb/ MagicId scales had a sensitivity of 63.89 , specificity of 85.87 , PPV of 77.97, and NPV of 75.24 for predicting SIPS positive group membership. These results suggest that classification of individuals into psychometric categories is a good indicator of whether an individual will report at least one clinically significant attenuated positive symptom. The high specificity suggests that most participants who do not have clinically significant symptoms will not meet criteria for positive schizotypy. However, the scales have relatively lower sensitivity in predicting SIPS scores, which suggests that some participants who have attenuated psychotic-like experiences may not be identified by the test. The high PPV suggests that most people who meet criteria for positive schizotypy will meet criteria for SIPS positive group membership, and the high NPV suggests that most participants who do not meet criteria for positive schizotypy will not report clinically significant positive symptoms.

Next, we conducted the same analyses for SocAnh diagnosing membership in the SIPS negative symptom group. The SocAnh had a sensitivity of 80.85 , specificity of 68.38 , PPV of 50.67 , and NPV of 89.89 . These results suggest that most participants who experience clinically significant negative symptoms will meet criteria for high negative schizotypy (sensitivity), and most participants who do not meet criteria for negative schizotypy will not report clinically significant negative symptoms (negative predictive value). The SocAnh exhibited lower specificity and positive predictive value than the PerAb and MagicId, which suggests that participants endorsed more anhedonia by questionnaire than was observed and rated in the interview.

To complement these sensitivity/specificity analyses, we conducted a receiver operating characteristic (ROC) curve analysis. A ROC analysis plots the true positive rate on the $y$-axis, which is equivalent to sensitivity, versus the false positive rate (i.e., 1-test specificity) on the $x$-axis. In addition to producing a graphical depiction of the data, ROC analyses allow us to estimate the area under the curve (AUC), which represents the accuracy of the test. In the current research, if the psychometric schizotypy scales were no better than chance at identifying people who have experienced psychotic-like symptoms, we could expect to see a perfect diagonal line in Figure 1 and Figure 2 (i.e., a perfect correlation between true positive rates and false positive rates) and an AUC of 0.5. Although there are no generally agreed upon criteria for what AUCs are "good" and "poor," some researchers have suggested the following: AUCs between .9 and 1 are excellent, between .8 and .89 are good, between .70 and .79 are fair, between .60 and 69 are poor, and less than .60 are useless (e.g., Sandelowsky, Stallberg, Nager, \& Hasselstrom, 2011).

As can be seen in Table 5, the MagicId, PerAb, and combination of the two all had high AUCs in predicting positive SIPS group membership that were significant at the $p<.001$ level, but the SocAnh did not. In contrast, the SocAnh was the only scale that significantly predicted SIPS negative group membership. The AUC can be interpreted as the probability that a person in the SIPS positive group would have a higher score on the schizotypy scale 


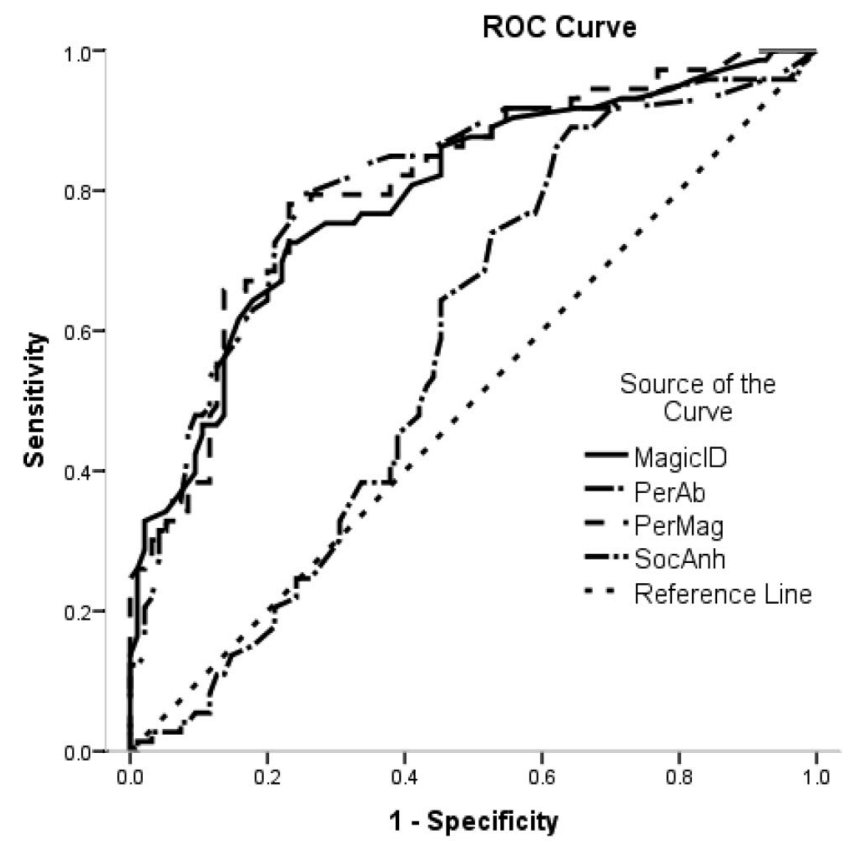

Diagonal segments are produced by ties.

Figure 1. Receiver operating characteristic (ROC) curve for the prediction of at least one Structured Interview for Prodromal Syndromes positive subscale score of 3 or greater. MagicID = Magical Ideation Scale; PerAb $=$ Perceptual Aberration Scale; PerMag = Perceptual Aberration/ Magical Ideation Scale; SocAnh $=$ Revised Social Anhedonia Scale.

than would a person in the SIPS control group. These findings suggest that self-report scores on the positive and negative schizotypy scales are a good approximation of whether individuals selected based on risk status report clinically meaningful positive and negative symptoms on an interview measure in a relatively small, mostly White, undergraduate sample.

\section{Discussion}

The overarching goal in the current research was to examine the correspondence between risk for schizophrenia measured with the psychometric and clinical high-risk approaches. The results suggest that the majority of participants identified as at risk with the self-report psychometric schizotypy approach (i.e., schizotypes) also report clinically meaningful attenuated positive symptoms on an interview measure in a relatively small, mostly White, undergraduate sample. Moreover, the schizotypy scales had good sensitivity and specificity in predicting which participants reported clinically significant positive and negative symptoms. However, results suggest that few of the people identified as at risk with the psychometric at-risk strategy in this sample meet criteria for attenuated psychosis syndrome, brief intermittent psychotic symptoms prodromal syndrome, or genetic risk and deterioration prodromal syndrome.

The results of the current research indicate that undergraduates identified as at risk with the psychometric approach indeed report experiencing meaningful attenuated positive symptoms. This lends credence to the utility of using schizotypy as a model for under- standing psychosis while removing confounds of patient research such as medication. On the SOPS, attenuated positive symptoms are conceptualized as ratings of 3 or greater on the positive rating scales. The current research found that $73 \%$ percent of the psychometric positive schizotypy group met this criterion for attenuated positive symptoms. These results are slightly higher than but generally consistent with previous research using the Wisconsin Manual, which found estimates of $59-60 \%$ for the PerAb group, $54 \%$ for the MagicId group, and $42-45 \%$ for the combined PerMag group (Allen et al., 1987; Chapman \& Chapman, 1980; Eckblad \& Chapman, 1983).

In addition to finding that the majority of people with psychometric positive schizotypy reported attenuated positive symptoms on the SOPS, the current research found that SOPS ratings and Wisconsin Schizotypy Scales scores were highly correlated. This provides a reference point for comparing results from psychometric schizotypy and clinical high-risk studies as well as convergent validity for both the Wisconsin Schizotypy Scales and SOPS ratings. This finding is consistent with previous research that has found that people at psychometric and clinical high risk have similar impairments in cognition (Brewer et al., 2006; Kerns \& Becker, 2008; Lenzenweger, Cornblatt, \& Putnick, 1991; Seidman et al., 2010), social cognition (e.g., Green et al., 2012; A. B. Miller \& Lenzenweger, 2012), and comorbid anxiety and depression symptoms (Lewandowski et al., 2006; Yung et al., 2007), among other deficits. The results of the current research suggest that there may be similarities between psychometric and clinical high-risk samples.

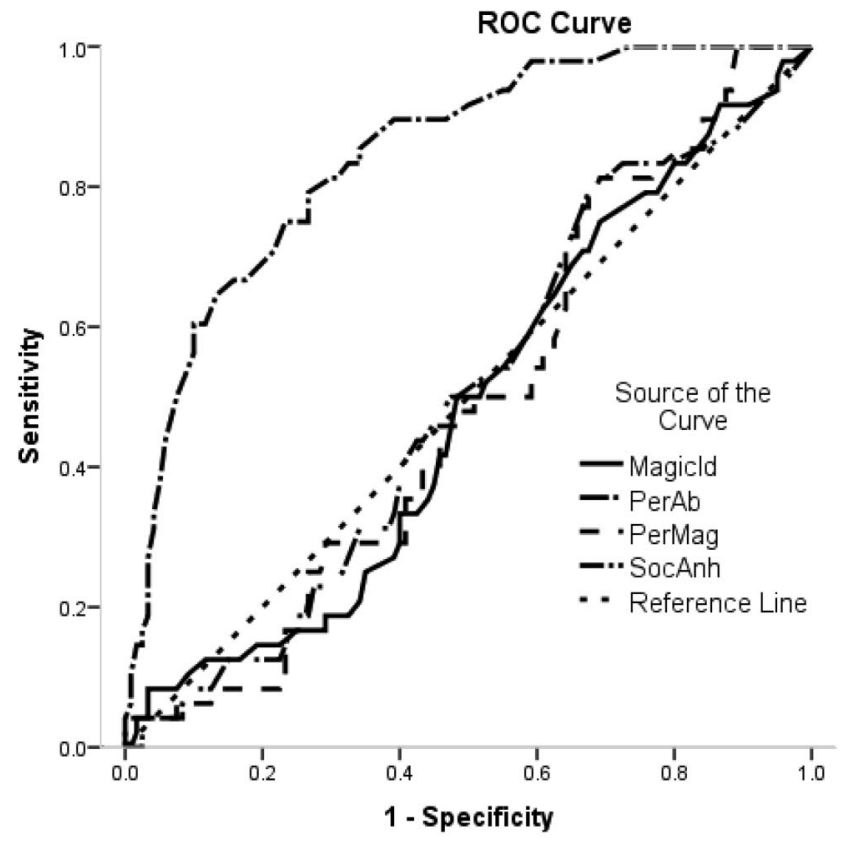

Diagonal segments are produced by ties.

Figure 2. Receiver operating characteristic (ROC) curve for the prediction of at least one Structured Interview for Prodromal Syndromes negative subscale score of 3 or greater. MagicID = Magical Ideation Scale; PerAb = Perceptual Aberration Scale; PerMag = Perceptual Aberration/ Magical Ideation Scale; SocAnh = Revised Social Anhedonia Scale. 
Table 5

Prediction of Positive and Negative SIPS Group Membership From Schizotypy Scales

\begin{tabular}{|c|c|c|c|c|c|c|}
\hline \multirow[b]{2}{*}{ Scale } & \multicolumn{3}{|c|}{ Positive SIPS } & \multicolumn{3}{|c|}{ Negative SIPS } \\
\hline & AUC $(S E)$ & $95 \% \mathrm{CI}$ & $p$ & $\begin{array}{c}\text { AUC } \\
(S E)\end{array}$ & $95 \%$ CI & $p$ \\
\hline Magical Ideation & $0.79(.04)$ & {$[0.72,0.86]$} & $<.001$ & $0.48(.05)$ & {$[0.39,0.58]$} & .736 \\
\hline Perceptual Aberration & $0.80(.04)$ & {$[0.73,0.87]$} & $<.001$ & $0.50(.05)$ & {$[0.41,0.89]$} & .986 \\
\hline Perceptual Aberration/Magical Ideation & $0.81(.03)$ & {$[0.74,0.88]$} & $<.001$ & $0.49(.05)$ & {$[0.40,0.58]$} & .851 \\
\hline Social Anhedonia & $0.58(.04)$ & {$[0.50,0.67]$} & .149 & $0.84(.03)$ & {$[0.78,0.91]$} & $<.001$ \\
\hline
\end{tabular}

Note. $\quad$ SIPS $=$ Structured Interview for Prodromal Syndromes; AUC $=$ area under the curve; $S E=$ standard error; $\mathrm{CI}=$ confidence interval.

One question raised by the current research is whether the level of risk is similar in undergraduates identified with the psychometric schizotypy approach and the clinical high-risk approach. The participants identified with the psychometric schizotypy approach clearly do not have the same imminent risk as participants identified by the clinical high-risk approach. Although $43 \%$ of the participants in the current research across all groups reported attenuated positive symptoms (i.e., SOPS ratings of 3 or higher on a positive symptom), only two participants met criteria for attenuated positive symptoms prodromal syndrome. The major difference between the current participants who experienced attenuated positive symptoms and participants meeting criteria for APSP were that most of these participants reported that the symptoms began more than 12 months prior to the study and that they had not worsened in the last 12 months. Moreover, many participants reported that the experiences were not as frequent as required by APSP criteria (i.e., once a week in the last month). This finding is consistent with previous research on attenuated positive symptoms in undergraduates, which suggests that $43 \%$ of college students report positive symptoms on the Prodromal Questionnaire, while only $25 \%$ report the symptoms at a high enough frequency, and only $2 \%$ report finding the symptoms to be distressing (Loewy, Johnson, \& Cannon, 2007). In the current research, two participants were also classified as at risk due to brief intermittent psychotic symptoms. However, no participants met criteria for genetic risk and deterioration prodromal syndrome. Although 22 participants met criteria for schizotypal personality disorder, no participants reported first-degree relatives with psychotic disorders and no participants reported a $30 \%$ decrease in global assessment of functioning in the last month prior to the study. This finding may be related to a relatively high global assessment of functioning necessary to remain enrolled in college. Potential participants meeting this category may have already left school and thus not been available to be sampled in the study. In contrast, participants in prodromal studies are often recruited through psychological clinics to which they are referred for help with existing problems. Thus, even the most severely schizotypal participants in the current study were unlikely to be bothered by their symptoms enough to seek help, whereas participants in prodromal studies are exclusively help-seeking individuals.

Another possible explanation for people identified in nonclinical settings as at risk not meeting risk categories according to the SOPS may be that psychometric risk represents an earlier point in the progression to the disorder (i.e., premorbid rather than prodromal). Meehl's original conceptualization of schizotypy was that it had a base rate of about $10 \%$ in the general population and that only $10 \%$ of people with schizotypy go on to develop full-blown schizophrenia (Meehl, 1962). Previous longitudinal studies have found that around 5\% of people identified as psychometric schizotypes with the current methodology develop a psychotic disorder at a 10-year follow up and that this rate increases to nearly $40 \%$ in people with both high negative and positive schizotypy (Chapman et al., 1994). In contrast, recent studies estimate that as many as $35 \%$ of people identified as at risk in the clinical high-risk study go on to convert to psychosis within two years (see Addington \& Heinssen, 2012, for a review). One explanation for these differences may be that participants in the clinical high-risk approach are at imminent risk for psychosis, but participants in the psychometric schizotypy approach have a more distal risk for psychotic disorders. In other words, participants identified as at risk with the psychometric schizotypy approach may still be in the premorbid phase, and participants in clinical high-risk studies are in the prodromal phase.

As mentioned, we chose undergraduates as research participants to enable comparisons between schizotypy research that is commonly done with undergraduates and clinical high-risk work. However, there are several notable limitations to undergraduate samples that limit the generalizability of the current results. Undergraduates may be higher functioning than samples drawn from the community or from outpatient clinics. Another potential difference between undergraduates and young adults is that undergraduates tend to have higher socioeconomic status by virtue of being able to afford college tuition, and they have more education than young adults not attending college. Thus, it is questionable if the current results would generalize to community or outpatient samples. At the same time, researchers have suggested that the use of undergraduates to model psychopathology can have both empirical and clinical value (Gotlib, 1984). Future research could examine the correspondence between psychometric and clinical risk for psychosis in community samples. Moreover, future research could use a similar study design with help-seeking individuals to test whether the results of the schizotypy scales correspond with the results of the SIPS in clinical samples.

Another limitation of the current research is that the sample was mostly White. Although the sample demographics are consistent with the demographics of the state from which it was drawn (U.S. Census, 2010), whether the results of the current research can be generalized to minority populations is unclear. Some previous research suggests that White participants have lower means on these scales than minority participants do (Chmielewski, Fer- 
nandes, Yee, \& Miller, 1995), and some items display differential item functioning between African American and White participants and between men and women (Winterstein et al., 2011). Winterstein et al. (2011) concluded that these scales should be revised and that subsample norms should be used. In the current research, we used different norms for men and women but did not use different norms for White and African American participants. Because African Americans tend to have higher scores, it is possible that some of the African Americans in our sample who were categorized as having a high psychometric risk for psychosis are false positives. If we used a higher mean for $z$-score calculations for African Americans, our sample would have included even fewer African Americans because some would have not met this higher score. The sample size of African Americans in the current research $(n=18)$ is too small for us to make meaningful comparisons between groups or to examine differential item functioning in these data. Future research including more diverse samples and appropriate norms could increase the generalizability of these results. As conducted, the findings can only be generalized to other mostly White, undergraduate populations.

Another potential limitation of the current research is that participants were not systematically assessed for cognition, social cognition, or Axis I psychopathology. It is possible that all three of these variables could have affected the results. For example, observed negative symptoms could have been related to depressed mood of participants rather than a risk for psychosis. Future research could examine the correspondence between these assessments and include a measure of functioning such as the Measurement and Treatment Research to Improve Cognition in Schizophrenia (MATRICS) Consensus Cognitive Battery (Nuechterlein et al., 2008) and an interview for Axis I psychopathology such as the Structured Clinical Interview for the DSM (First, Spitzer, Gibbon, \& Williams, 1998).

In the current research, the interviewer was blind to the risk status of the participants (i.e., he did not know whether the participants met criteria for the positive schizotypy, negative schizotypy, or comparison group). However, the interviewer knew that the study design indicated approximately one third of the participants would meet criteria for positive schizotypy and one third would meet criteria for negative schizotypy. Thus, two thirds of the sample was expected to report clinically meaningful symptoms, which is much higher than would be expected if participants were randomly drawn from the community. We chose this psychometric high-risk approach because it is a commonly used strategy to assess low base-rate phenomena. This approach may have resulted in elevated estimates of congruence between the two methods. However, only four participants from the comparison group were rated as having 3 or higher on any positive rating. If the experimenter systematically overrated attenuated psychotic symptoms, we would expect to find higher rates of "false positives" in the comparison group.

In the current research, we interpreted the high correlations between the schizotypy scales and the SOPS ratings to be evidence of the convergent validity of schizotypy scale scores with SOPS ratings. Our interpretation of the convergent validity of scale scores is grounded in a construct validity approach suggesting that, in the absence of a true gold standard, researchers need to validate scale scores by examining the convergent validity of scales with measures of other constructs in its "nomological network" (Cron- bach \& Meehl, 1955). In a nomological network, a pattern of correlations among relevant constructs is hypothesized and then systematically tested. Often, researchers use self-report measures of similar constructs or self-report and interview measures of the same or related construct (e.g., schizotypy and prodromal symptoms in the current research). Interviews offer several advantages over self-report measures, such as basing ratings on observations in addition to participant answers, allowing for follow-up questions, and enabling both the interviewee and interviewer to clarify questions and responses (Sher \& Trull, 1996). For example, many SOPS ratings are based on observations (e.g., disorganized communication, expression of emotion, odd behavior or appearance), and all ratings are based on follow-up questions about onset, duration, distress, and conviction of beliefs. However, it should be noted that interviews can largely involve self-report, and there may be substantial criterion overlap between the self-report and interview measures. In the current research, participants self-reported on a questionnaire about their psychotic-like experiences and then self-reported in an interview about their psychotic-like experiences. An alternative interpretation of these results could be temporal stability or test-retest reliability. In this interpretation, it could be concluded that schizotypy scale scores have moderate temporal stability in assessing attenuated psychotic symptoms.

In addition to differences between schizotypy and prodromal constructs, one explanation for the differences in the two approaches could be related to general differences in self-report and interview assessments. In the current research, we chose the SIPS as the interview measure because it is the most commonly used interview measure of psychosis proneness in North America and is the measure used in the North American Prodrome Longitudinal Study (Addington et al., 2007; 2012). Future research could examine the correspondence between schizotypy scales and other interview measures of psychosis risk, such as the Comprehensive Assessment of At-Risk Mental States (Raballo, Nelson, Thompson, \& Yung, 2011; Yung et al., 2005), the Bonn Scale for the Assessment of Basic Symptoms (BSABS; Gross, 1989; Klosterkötter et al., 2001), or the Schizophrenia Proneness Instrument for Adults (SPI-A; Schultze-Lutter, Klosterkötter, Picker, Steinmeyer, \& Ruhrmann, 2007). Future research exploring the correspondence of the schizotypy scales and the BSABS/SPI-A could be important because both approaches aim to identify people at risk at very early or premorbid stages of the illness, potentially before people seek help (Klosterkötter, Schultze-Lutter, \& Ruhrmann, 2008; Lenzenweger, 1994; Schultze-Lutter, 2009).

As mentioned, some previous research has attempted to validate the schizotypy scale scores by comparing them to interview-based measures of subclinical psychotic-like experiences (Gross, Silvia, Barrantes-Vidal, \& Kwapil, 2012). Additionally, other research has attempted to develop interview measures of schizotypy itself. For example, the authors of the schizotypy scales developed and validated the Wisconsin Manual for Assessing Psychotic-Like Experiences (Chapman \& Chapman, 1980; Kwapil et al., 1999). In addition, the Structured Interview for Schizotypy (Kendler, Lieberman, \& Walsh, 1989), the Symptom Schedule for the Diagnosis of Borderline Schizophrenia (Khouri, Haier, Rieder, \& Rosenthal, 1980), and the Schedule for Schizotypal Personalities (Baron, Asnis, \& Gruen, 1981) all measure schizotypy. Although the correspondence between schizotypy and interview-rated, psychotic-like experiences was good in the current study with 
undergraduates, future research could examine whether interviewrated schizotypy has an even higher correspondence with prodromal interviews like the SIPS. Taken together, this future research would help to elucidate whether the differences between schizotypy scales and prodromal interviews are due to differences between the prodromal and schizotypy constructs, to the different methods (i.e., interview vs. self-report questionnaire), or to some combination of the two.

Although one unique aspect of the current research is that it is the first to compare the Wisconsin Schizotypy Scales and the SIPS in a study, previous research has used self-report psychosis risk screening measures and the SIPS. For example, researchers have administered the Prime Screen, Prodromal Questionnaire-Brief, and Youth Psychosis At-Risk Questionnaire-Brief along with the SIPS and found high correlations among the measures (Kline et al., 2012). Recent research has found that the Prodromal Questionnaire (Loewy, Bearden, Johnson, Raine, \& Cannon, 2005; Loewy, Pearson, Vinogradov, Bearden, \& Cannon, 2011), a combination of Schizotypal Personality Questionnaire items and the probes from the SIPS, can be used with $91 \%$ sensitivity and $49 \%$ specificity to diagnose people as prodromal in help-seeking individuals referred to a prodromal specialty clinic. However, these screening instruments were specifically designed to be convergent with psychosis risk interviews, and few have been used with the undergraduate samples that are common in schizotypy studies. The current research extends this previous work by using the Wisconsin Schizotypy Scales in a sample commonly used in schizotypy research. Future research could examine the correlations between the schizotypy scales and prodromal screens to further clarify whether the observed difference in the current research are due to method variance (i.e., interview vs. self-report) or to differences in latent constructs (i.e., schizotypy vs. prodrome).

The clinical high-risk approach to the assessment of risk for psychosis focuses mainly on the positive symptoms of psychosis. Negative and disorganized symptoms are assessed but do not factor into psychosis risk categories. Previous research suggests that persistent negative symptoms are related to a longer duration of psychosis (Chang et al., 2011; Galderisi et al., 2013) and worse prognosis (Boonstra et al., 2012; Díaz et al., 2013). Moreover, some research has found that DUP is associated with negative symptom severity, but not with positive symptom severity (de Haan, van Der Gaag, \& Wolthaus, 2000), and that interventions aimed to shorten DUP may lessen the impact of negative symptoms (Melle et al., 2008). In general, negative symptoms have been understudied in risk for psychosis research despite having an earlier onset than positive symptoms, being associated with conversion to psychosis, and being related to worse prognosis (Pelletier \& Mittal, 2013). Future research could continue to examine the role of negative symptoms in duration of untreated psychosis and severity of illness.

In the current research, the SocAnh displayed lower levels of specificity than the PerAb and MagicId. This suggests that the SocAnh is less specific in predicting negative symptoms as measured with the SIPS than the positive schizotypy scales are in predicting positive symptoms on the SIPS with the current cut scores. When choosing a cut score it is important to weigh the risks and benefits of false positives and negatives. In the clinical highrisk approach, it may be especially harmful to have false negatives because an individual in need of treatment may not receive it.
However, because schizotypy is primarily a research tool, researchers may want to emphasize eliminating false positives because the presence of false positives in a schizotypy group may obscure significant results. Thus, researchers may want to consider using a higher cut score than 1.96 SDs above the mean on the SocAnh to create negative schizotypy groups. This would increase the specificity of SocAnh in predicting interview-rated negative symptoms while leaving the sensitivity at acceptable levels.

\section{References}

Addington, J., Cadenhead, K. S., Cannon, T. D., Cornblatt, B., McGlashan, T. H., Perkins, D. O., . . Heinssen, R. (2007). North American Prodrome Longitudinal Study: A collaborative multisite approach to prodromal schizophrenia research. Schizophrenia Bulletin, 33, 665-672. doi:10.1093/schbul/sb1075

Addington, J., Cadenhead, K. S., Cornblatt, B. A., Mathalon, D. H., McGlashan, T. H., Perkins, D. O., . . Cannon, T. D. (2012). North American Prodrome Longitudinal Study (NAPLS 2): Overview and recruitment. Schizophrenia Research, 142, 77-82. doi:10.1016/j.schres .2012 .09 .012

Addington, J., Cornblatt, B. A., Cadenhead, K. S., Cannon, T. D., McGlashan, T. H., Perkins, D. O., . . Heinssen, R. (2011). At clinical high risk for psychosis: Outcome for nonconverters. American Journal of Psychiatry, 168, 800-805. doi:10.1176/appi.ajp.2011.10081191

Addington, J., Epstein, I., Liu, L., French, P., Boydell, K. M., \& Zipursky, R. B. (2011). A randomized controlled trial of cognitive behavioral therapy for individuals at clinical high risk of psychosis. Schizophrenia Research, 125, 54-61. doi:10.1016/j.schres.2010.10.015

Addington, J., \& Heinssen, R. (2012). Prediction and prevention of psychosis in youth at clinical high risk. Annual Review of Clinical Psychology, 8, 269-289. doi:10.1146/annurev-clinpsy-032511-143146

Addington, J., Leriger, E., \& Addington, D. (2003). Symptom outcome 1 year after admission to an early psychosis program. Canadian Journal of Psychiatry/Revue canadienne de psychiatrie, 48, 204-207.

Allen, J. J., Chapman, L. J., Chapman, J. P., Vuchetich, J. P., \& Frost, L. A. (1987). Prediction of psychoticlike symptoms in hypothetically psychosis-prone college students. Journal of Abnormal Psychology, 96, 83-88. doi:10.1037/0021-843X.96.2.83

American Psychiatric Association. (2013). Diagnostic and statistical manual of mental disorders (5th ed.). Arlington, VA: American Psychiatric Publishing.

Baron, M., Asnis, L., \& Gruen, R. (1981). The Schedule for Schizotypal Personalities (SSP): A diagnostic interview for schizotypal features. Psychiatry Research, 4, 213-228. doi:10.1016/0165-1781(81)90024-X

Boonstra, N., Klaassen, R., Sytema, S., Marshall, M., De Haan, L., Wunderink, L., \& Wiersma, D. (2012). Duration of untreated psychosis and negative symptoms-A systematic review and meta-analysis of individual patient data. Schizophrenia Research, 142, 12-19. doi:10.1016/j .schres.2012.08.017

Brewer, W. J., Wood, S. J., Phillips, L. J., Francey, S. M., Pantelis, C., Yung, A. R., . . . McGorry, P. D. (2006). Generalized and specific cognitive performance in clinical high-risk cohorts: A review highlighting potential vulnerability markers for psychosis. Schizophrenia Bulletin, 32, 538-555. doi:10.1093/schbul/sbj077

Cannon, T. D., Cadenhead, K., Cornblatt, B., Woods, S. W., Addington, J., Walker, E., . . Heinssen, R. (2008). Prediction of psychosis in youth at high clinical risk: A multisite longitudinal study in North America. Archives of General Psychiatry, 65, 28-37. doi:10.1001/archgenpsychiatry.2007.3

Chang, W. C., Hui, C. L., Tang, J. Y., Wong, G. H., Lam, M. M., Chan, S. K., \& Chen, E. Y. (2011). Persistent negative symptoms in firstepisode schizophrenia: A prospective three-year follow-up study. Schizophrenia Research, 133, 22-28. doi:10.1016/j.schres.2011.09.006 
Chapman, L. J., \& Chapman, J. P. (1980). Scales for rating psychotic and psychotic-like experiences as continua. Schizophrenia Bulletin, 6, 476489. doi:10.1093/schbul/6.3.476

Chapman, L. J., Chapman, J. P., Kwapil, T. R., Eckblad, M., \& Zinser, M. C. (1994). Putatively psychosis-prone subjects 10 years later. Journal of Abnormal Psychology, 103, 171-183. doi:10.1037/0021-843X.103.2 .171

Chapman, L. J., Chapman, J. P., \& Raulin, M. L. (1978). Body-image aberration in schizophrenia. Journal of Abnormal Psychology, 87, 399407. doi:10.1037/0021-843X.87.4.399

Chapman, L. J., Chapman, J. P., Raulin, M. L., \& Edell, W. S. (1978). Schizotypy and thought disorder as a high risk approach to schizophrenia. In G. Serban (Ed.), Cognitive defects in the development of mental illness (pp. 351-360). New York, NY: Brunner/Mazel.

Chmielewski, P. M., Fernandes, L. O., Yee, C. M., \& Miller, G. A. (1995). Ethnicity and gender in scales of psychosis proneness and mood disorders. Journal of Abnormal Psychology, 104, 464-470. doi:10.1037/ 0021-843X.104.3.464

Cicero, D. C., \& Kerns, J. G. (2010). Multidimensional factor structure of positive schizotypy. Journal of Personality Disorders, 24, 327-343. doi:10.1521/pedi.2010.24.3.327

Cronbach, L. J., \& Meehl, P. E. (1955). Construct validity in psychological tests. Psychological Bulletin, 52, 281-302. doi:10.1037/h0040957

de Haan, L., van Der Gaag, M., \& Wolthaus, J. (2000). Duration of untreated psychosis and the long-term course of schizophrenia. European Psychiatry, 15, 264-267. doi:10.1016/S0924-9338(00)00234-0

Delawalla, Z., Barch, D. M., Fisher Eastep, J. L., Thomason, E. S., Hanewinkel, M. J., Thompson, P. A., \& Csernansky, J. G. (2006). Factors mediating cognitive deficits and psychopathology among siblings of individuals with schizophrenia. Schizophrenia Bulletin, 32, 525-537. doi:10.1093/schbul/sbj082

Díaz, I., Pelayo-Terán, J. M., Pérez-Iglesias, R., Mata, I., TabarésSeisdedos, R., Suárez-Pinilla, P., . . C Crespo-Facorro, B. (2013). Predictors of clinical remission following a first episode of non-affective psychosis: Sociodemographics, premorbid and clinical variables. Psychiatry Research, 206, 181-187. doi:10.1016/j.psychres.2012.10.011

Eckblad, M., \& Chapman, L. J. (1983). Magical ideation as an indicator of schizotypy. Journal of Consulting and Clinical Psychology, 51, 215225. doi:10.1037/0022-006X.51.2.215

Eckblad, M., Chapman, L. J., Chapman, J. P., \& Mishlove, M. (1982). The revised social anhedonia scale. (Available from L. J. Chapman, Department of Psychology, 1202 West Johnson Street, University of Wisconsin, Madison, WI 53706).

Edell, W. S. (1995). The psychometric measurement of schizotypy using the Wisconsin Scales of Psychosis Proneness. In G. Miller (Ed.), The behavioral high-risk paradigm in psychopathology (pp. 1-46). New York, NY: Springer-Verlag.

First, M. B., Spitzer, R. L., Gibbon, M., \& Williams, J. B. W. (1998). Structured Clinical Interview for DSM-IV Axis I Disorders. New York, NY: New York State Psychiatric Institute.

Fonseca-Pedrero, E., Paíno, M., Lemos-Giráldez, S., García-Cueto, E., Campillo-Alvarez, A., Villazón-Garcia, U., \& Muñiz, J. (2008). Schizotypy assessment: State of the art and future prospects. International Journal of Clinical and Health Psychology, 8, 577-593.

Galderisi, S., Mucci, A., Bitter, I., Libiger, J., Bucci, P., \& Fleischhacker, W. W. (2013). Persistent negative symptoms in first episode patients with schizophrenia: Results from the European First Episode Schizophrenia Trial. European Neuropsychopharmacology, 23, 196-204. doi: 10.1016/j.euroneuro.2012.04.019

Gooding, D. C., Tallent, K. A., \& Matts, C. W. (2005). Clinical status of at-risk individuals 5 years later: Further validation of the psychometric high-risk strategy. Journal of Abnormal Psychology, 114, 170-175. doi:10.1037/0021-843X.114.1.170
Gotlib, I. H. (1984). Depression and general psychopathology in university students. Journal of Abnormal Psychology, 93, 19-30. doi:10.1037/ 0021-843X.93.1.19

Graves, R. E., \& Weinstein, S. (2004). A Rasch analysis of three of the Wisconsin Scales of Psychosis Proneness: Measurement of schizotypy. Journal of Applied Measurement, 5, 160-171.

Green, M. F., Bearden, C. E., Cannon, T. D., Fiske, A. P., Hellemann, G. S., Horan, W. P., . . Nuechterlein, K. H. (2012). Social cognition in schizophrenia, Part 1: Performance across phase of illness. Schizophre nia Bulletin, 38, 854-864. doi:10.1093/schbul/sbq171

Gross, G. (1989). The basic symptoms of schizophrenia. British Journal of Psychiatry, 155(Suppl. 7), 21-25.

Gross, G. M., Silvia, P. J., Barrantes-Vidal, N., \& Kwapil, T. R. (2012). Psychometric properties and validity of short forms of the Wisconsin Schizotypy Scales in two large samples. Schizophrenia Research, 134, 267-272. doi:10.1016/j.schres.2011.11.032

Haynes, S. N., Smith, G. T., \& Hunsley, J. D. (2011). Scientific foundations of clinical assessment. New York, NY: Routledge.

Horan, W. P., Brown, S. A., \& Blanchard, J. J. (2007). Social anhedonia and schizotypy: The contribution of individual differences in affective traits, stress, and coping. Psychiatry Research, 149, 147-156. doi: 10.1016/j.psychres.2006.06.002

Kay, S. R., Fiszbein, A., \& Opler, L. A. (1987). The Positive and Negative Syndrome Scale (PANSS) for schizophrenia. Schizophrenia Bulletin, 13, 261-276. doi:10.1093/schbul/13.2.261

Kendler, K. S., Lieberman, J. A., \& Walsh, D. (1989). The Structured Interview for Schizotypy (SIS): A preliminary report. Schizophrenia Bulletin, 15, 559-571. doi:10.1093/schbul/15.4.559

Kendler, K. S., Tsuang, M. T., \& Hays, P. (1987). Age at onset in schizophrenia: A familial perspective. Archives of General Psychiatry, 44, 881-890. doi:10.1001/archpsyc.1987.01800220047008

Kerns, J. G., \& Becker, T. M. (2008). Communication disturbances, working memory, and emotion in people with elevated disorganized schizotypy. Schizophrenia Research, 100, 172-180. doi:10.1016/j.schres .2007.11.005

Kerns, J. G., \& Berenbaum, H. (2003). The relationship between formal thought disorder and executive functioning component processes. Journal of Abnormal Psychology, 112, 339-352. doi:10.1037/0021-843X 112.3.339

Keshavan, M. S., Eack, S. M., Montrose, D. M., Abela, M. M., Bangalore, S. S., Diwadkar, V. A., . . . Rajarethinam, R. (2009). Do premorbid impairments predict emergent "prodromal" symptoms in young relatives at risk for schizophrenia? Early Intervention in Psychiatry, 3, 213-220. doi:10.1111/j.1751-7893.2009.00135.x

Khouri, P. J., Haier, R. J., Rieder, R. O., \& Rosenthal, D. (1980). A symptom schedule for the diagnosis of borderline schizophrenia: A first report. British Journal of Psychiatry, 137, 140-147. doi:10.1192/bjp 137.2.140

Kline, E., Wilson, C., Ereshefsky, S., Denenny, D., Thompson, E., Pitts, S. C., . . S Schiffman, J. (2012). Psychosis risk screening in youth: A validation study of three self-report measures of attenuated psychosis symptoms. Schizophrenia Research, 141, 72-77. doi:10.1016/j.schres 2012.07.022

Klosterkötter, J., Hellmich, M., Steinmeyer, E. M., \& Schultze-Lutter, F. (2001). Diagnosing schizophrenia in the initial prodromal phase. Archives of General Psychiatry, 58, 158-164. doi:10.1001/archpsyc.58.2 .158

Klosterkötter, J., Schultze-Lutter, F., \& Ruhrmann, S. (2008). Kraepelin and psychotic prodromal conditions. European Archives of Psychiatry and Clinical Neuroscience, 258(Suppl. 2), 74-84. doi:10.1007/s00406008-2010-5

Kwapil, T. R. (1998). Social anhedonia as a predictor of the development of schizophrenia-spectrum disorders. Journal of Abnormal Psychology, 107, 558-565. doi:10.1037/0021-843X.107.4.558 
Kwapil, T. R., Barrantes-Vidal, N., \& Silvia, P. J. (2008). The dimensional structure of the Wisconsin Schizotypy Scales: Factor identification and construct validity. Schizophrenia Bulletin, 34, 444-457. doi:10.1093/ schbul/sbm098

Kwapil, T. R., Chapman, L. J., \& Chapman, J. (1999). Validity and usefulness of the Wisconsin Manual for Assessing Psychotic-like Experiences. Schizophrenia Bulletin, 25, 363-375. doi:10.1093/oxfordjournals.schbul.a033384

Kwapil, T. R., Miller, M. B., Zinser, M. C., Chapman, J., \& Chapman, L. J. (1997). Magical ideation and social anhedonia as predictors of psychosis proneness: A partial replication. Journal of Abnormal Psychology, 106, 491-495. doi:10.1037/0021-843X.106.3.491

Lenzenweger, M. F. (1994). Psychometric high-risk paradigm, perceptual aberrations, and schizotypy: An update. Schizophrenia Bulletin, 20, 121-135. doi:10.1093/schbul/20.1.121

Lenzenweger, M. F., Cornblatt, B. A., \& Putnick, M. (1991). Schizotypy and sustained attention. Journal of Abnormal Psychology, 100, 84-89. doi:10.1037/0021-843X.100.1.84

Levine, S. Z., \& Rabinowitz, J. (2009). A population-based examination of the role of years of education, age of onset, and sex on the course of schizophrenia. Psychiatry Research, 168, 11-17. doi:10.1016/j.psychres .2008 .05 .005

Lewandowski, K. E., Barrantes-Vidal, N., Nelson-Gray, R. O., Clancy, C., Kepley, H. O., \& Kwapil, T. R. (2006). Anxiety and depression symptoms in psychometrically identified schizotypy. Schizophrenia Research, 83, 225-235. doi:10.1016/j.schres.2005.11.024

Loewy, R. L., Bearden, C. E., Johnson, J. K., Raine, A., \& Cannon, T. D. (2005). The Prodromal Questionnaire (PQ): Preliminary validation of a self-report screening measure for prodromal and psychotic syndromes. Schizophrenia Research, 79, 117-125. doi:10.1016/j.schres.2005.03.007

Loewy, R. L., Johnson, J. K., \& Cannon, T. D. (2007). Self-report of attenuated psychotic experiences in a college population. Schizophrenia Research, 93, 144-151. doi:10.1016/j.schres.2007.02.010

Loewy, R. L., Pearson, R., Vinogradov, S., Bearden, C. E., \& Cannon, T. D. (2011). Psychosis risk screening with the Prodromal Questionnaire-Brief Version (PQ-B). Schizophrenia Research, 129, 4246. doi:10.1016/j.schres.2011.03.029

Marshall, M., Lewis, S., Lockwood, A., Drake, R., Jones, P., \& Croudace, T. (2005). Association between duration of untreated psychosis and outcome in cohorts of first-episode patients: A systematic review. Archives of General Psychiatry, 62, 975-983. doi:10.1001/archpsyc.62.9 .975

Meehl, P. E. (1962). Schizotaxia, schizotypy, and schizophrenia. American Psychologist, 17, 827-838. doi:10.1037/h0041029

Melle, I., Larsen, T. K., Haahr, U., Friis, S., Johannesen, J. O., Opjordsmoen, S., . . McGlashan, T. (2008). Prevention of negative symptom psychopathologies in first-episode schizophrenia: Two-year effects of reducing the duration of untreated psychosis. Archives of General Psychiatry, 65, 634-640. doi:10.1001/archpsyc.65.6.634

Miller, A. B., \& Lenzenweger, M. F. (2012). Schizotypy, social cognition, and interpersonal sensitivity. Personality Disorders: Theory, Research, and Treatment, 3, 379-392. doi:10.1037/a0027955

Miller, T. J., McGlashan, T. H., Rosen, J. L., Cadenhead, K., Cannon, T., Ventura, J., . . Woods, S. W. (2003). Prodromal assessment with the Structured Interview for Prodromal Syndromes and the Scale of Prodromal Symptoms: Predictive validity, interrater reliability, and training to reliability. Schizophrenia Bulletin, 29, 703-715. doi:10.1093/ oxfordjournals.schbul.a007040

Miller, T. J., McGlashan, T. H., Rosen, J. L., Somjee, L., Markovich, P. J., Stein, K., \& Woods, S. W. (2002). Prospective diagnosis of the initial prodrome for schizophrenia based on the Structured Interview for Prodromal Syndromes: Preliminary evidence of interrater reliability and predictive validity. American Journal of Psychiatry, 159, 863-865. doi:10.1176/appi.ajp.159.5.863
Neale, J. M., \& Oltmanns, T. F. (1980). Schizophrenia. New York, NY: Wiley.

Norman, R. M., Lewis, S. W., \& Marshall, M. (2005). Duration of untreated psychosis and its relationship to clinical outcome. British Journal of Psychiatry Supplement, 48, s19-s23. doi:10.1192/bjp.187.48 .s19

Nuechterlein, K. H., Green, M. F., Kern, R. S., Baade, L. E., Barch, D. M., Cohen, J. D., . . Marder, S. R. (2008). The MATRICS Consensus Cognitive Battery, Part 1: Test selection, reliability, and validity. American Journal of Psychiatry, 165, 203-213. doi:10.1176/appi.ajp.2007 .07010042

Pelletier, A. L., \& Mittal, V. A. (2013). Negative symptom measurement in individuals at-risk for psychosis. Psychiatry Research, 205, 181-182. doi:10.1016/j.psychres.2012.08.020

Raballo, A., Nelson, B., Thompson, A., \& Yung, A. (2011). The comprehensive assessment of at-risk mental states: From mapping the onset to mapping the structure. Schizophrenia Research, 127, 107-114.

Rabinowitz, J., Levine, S. Z., \& Hafner, H. (2006). A population based elaboration of the role of age of onset on the course of schizophrenia. Schizophrenia Research, 88, 96-101. doi:10.1016/j.schres.2006.07.007

Sandelowsky, H., Stallberg, B., Nager, A., \& Hasselström, J. (2011). The prevalence of undiagnosed chronic obstructive pulmonary disease in a primary care population with respiratory tract infections-A case finding study. BMC Family Practice, 12, Article 122. doi:10.1186/14712296-12-122

Schultze-Lutter, F. (2009). Subjective symptoms of schizophrenia in research and the clinic: The basic symptom concept. Schizophrenia Bulletin, 35, 5-8. doi:10.1093/schbul/sbn139

Schultze-Lutter, F., Klosterkötter, J., Picker, H., Steinmeyer, E. M., \& Ruhrmann, S. (2007). Predicting first-episode psychosis by basic symptom criteria. Clinical Neuropsychiatry, 4, 11-22.

Seidman, L. J., Giuliano, A. J., Meyer, E. C., Addington, J., Cadenhead, K. S., Cannon, T. D., . . Cornblatt, B. A. (2010). Neuropsychology of the prodrome to psychosis in the NAPLS consortium: Relationship to family history and conversion to psychosis. Archives of General Psychiatry, 67, 578-588. doi:10.1001/archgenpsychiatry.2010.66

Sher, K. J., \& Trull, T. J. (1996). Methodological issues in psychopathology research. Annual Review of Psychology, 47, 371-400. doi:10.1146/ annurev.psych.47.1.371

Stoffelmayr, B. E., Dillavou, D., \& Hunter, J. E. (1983). Premorbid functioning and outcome in schizophrenia: A cumulative analysis. Journal of Consulting and Clinical Psychology, 51, 338-352. doi:10.1037/ 0022-006X.51.3.338

Tandon, N., Montrose, D., Shah, J., Rajarethinam, R. P., Diwadkar, V. A., \& Keshavan, M. S. (2012). Early prodromal symptoms can predict future psychosis in familial high-risk youth. Journal of Psychiatric Research, 46, 105-110. doi:10.1016/j.jpsychires.2011.09.019

Tandon, N., Shah, J., Keshavan, M. S., \& Tandon, R. (2012). Attenuated psychosis and the schizophrenia prodrome: Current status of risk identification and psychosis prevention. Neuropsychiatry, 2, 345-353. doi: 10.2217/npy. 12.36

U.S. Census Bureau. (2010). State \& county quickfacts: Missouri. Retrieved March 18, 2014, from http://quickfacts.census.gov/qfd/states/ 29000.html

Winterstein, B. P., Ackerman, T. A., Silvia, P. J., \& Kwapil, T. R. (2011). Psychometric properties of the Wisconsin Schizotypy Scales in an undergraduate sample: Classical test theory, item response theory, and differential item functioning. Journal of Psychopathology and Behavioral Assessment, 33, 480-490. doi:10.1007/s10862-011-9242-9

Woods, S. W., Walsh, B. C., Saksa, J. R., \& McGlashan, T. H. (2010). The case for including attenuated psychotic symptoms syndrome in DSM-5 as a psychosis risk syndrome. Schizophrenia Research, 123, 199-207. doi:10.1016/j.schres.2010.08.012 
Wuthrich, V. M., \& Bates, T. C. (2006). Confirmatory factor analysis of the three-factor structure of the Schizotypal Personality Questionnaire and Chapman Schizotypy Scales. Journal of Personality Assessment, 87, 292-304. doi:10.1207/s15327752jpa8703_10

Yung, A. R. (2003). Commentary: The schizophrenia prodrome: A highrisk concept. Schizophrenia Bulletin, 29, 859-865. doi:10.1093/ oxfordjournals.schbul.a007052

Yung, A. R., Buckby, J. A., Cosgrave, E. M., Killackey, E. J., Baker, K., Cotton, S. M., \& McGorry, P. D. (2007). Association between psychotic experiences and depression in a clinical sample over 6 months. Schizophrenia Research, 91, 246-253. doi:10.1016/j.schres 2006.11 .026
Yung, A. R., \& McGorry, P. D. (1996). The prodromal phase of firstepisode psychosis: Past and current conceptualizations. Schizophrenia Bulletin, 22, 353-370. doi:10.1093/schbul/22.2.353

Yung, A. R., Yuen, H. P., McGorry, P. D., Phillips, L. J., Kelly, D., Dell'Olio, M., . . Buckby, J. (2005). Mapping the onset of psychosis: The Comprehensive Assessment of At-Risk Mental States. Australia and New Zealand Journal of Psychiatry, 39, 964-971.

Received November 16, 2012 Revision received February 3, 2014 Accepted February 10, 2014

\section{Call for Submissions: Psychological Science in MedEdPORTAL Publications}

In recognition of the importance of psychology as both a basic and clinical science in the preparation of the health care workforce, Barney Beins, PhD (Associate Editor, MedEdPORTAL) is now soliciting submissions related to psychological science as part of a collaboration between the American Psychological Association and the Association of American Medical Colleges to create an online-only collection of free, peer-reviewed educational resources in MedEdPORTAL Publications. This repository is used across the health professions in the preparation of future practitioners.

Accepted submissions are complete stand-alone learning or teaching modules that have been successfully implemented in the classroom or clinical training environment. This effort is to collect case studies of pedagogical approaches to promote the scholarship of teaching and learning. See the MedEdPORTAL Publications site (https://www.mededportal.org/) for current examples and instructions for submission. We especially encourage submission of resources that facilitate the teaching of foundational psychological science related to behavior and health. Sample topics are listed below, but submissions are not limited to these areas.

- Social, emotional, and cognitive development

- Memory

- Perception

- Psychophysiology

- Psychoneuroimmunology

- Interpersonal relationships

- Behavior change

- Motivation

- Decision making

- Leadership

- Unconscious bias

- Group dynamics and team functioning
- Violence

- Psychometrics

- Stress and coping

- Treatment adherence

- Grief

- Behavioral health risk factors

- Obesity and weight management

- Smoking cessation

- Mental and behavioral disorders

- Health belief models

- Dental anxiety

- Pain

- Provider-patient communication 